\title{
Phronesis
}

\section{Accompagner un moment de transition professionnelle par un dispositif de formation-action réflexif mené auprès de conseillers en orientation et en accompagnement professionnels}

\section{To accompany one moment of professional transition with a reflexive device of formation-action carried out near accompaniment professionals and careers advisers}

\section{Martine Poulin}

Volume 4, numéro 4, 2016

Les voies et les voix de l'accompagnement

URI : https://id.erudit.org/iderudit/1036711ar

DOI : https://doi.org/10.7202/1036711ar

Aller au sommaire du numéro

Éditeur(s)

Université de Sherbrooke

ISSN

1925-4873 (numérique)

Découvrir la revue

Citer cet article

Poulin, M. (2016). Accompagner un moment de transition professionnelle par un dispositif de formation-action réflexif mené auprès de conseillers en orientation et en accompagnement professionnels. Phronesis, 4(4), 27-45. https://doi.org/10.7202/1036711ar

\section{Résumé de l'article}

La question de la professionnalisation des acteurs fait souvent débat, car elle pourrait sous-entendre qu'un acteur qui se professionnalise n'est pas un bon professionnel voire qu'il n'est pas compétent. Cette position est d'autant plus renforcée quand ces acteurs vivent des moments de transition professionnelle, où les doutes et incertitudes viennent bousculer leur identité professionnelle. Comment dès lors les accompagner au mieux dans ces moments qui questionnement?

Cet article vise à montrer comment par un dispositif de formation-action réflexif une démarche de professionnalisation peut être, d'une part un processus de construction des sujets leur permettant de prendre conscience de leur bagage d'expérience, de leur professionnalité et, d'autre part un processus contribuant à la construction de leur posture d'apprenance, de sujet apprenant. Il s'agit d'un processus en mouvement qui s'inscrit dans la philosophie de la formation et l'orientation tout au long de la vie, dans lequel le sujet doit se reconnaître comme un acteur au travail, comme un auteur de son travail et comme un sujet en travail sur son activité constructive favorisant ainsi son développement. 


\title{
Accompagner un moment de transition professionnelle par un dispositif de for- mation-action réflexif mené auprès de conseillers en orientation et en accompa- gnement professionnels
}

\author{
Martine POULIN
}

Centre de recherche sur le travail et le développement

CRDT, CNAM.

41, rue Gay-Lussac, 75005 Paris

martine.poulin@cnam.fr

Mots clés : Apprenance — Réflexivité - Soi - Conseil — Transition professionnelle — Professionnalisation — Expérience

Résumé : La question de la professionnalisation des acteurs fait souvent débat, car elle pourrait sous-entendre qu'un acteur qui se professionnalise n'est pas un bon professionnel voire qu'il n'est pas compétent. Cette position est d'autant plus renforcée quand ces acteurs vivent des moments de transition professionnelle, où les doutes et incertitudes viennent bousculer leur identité professionnelle. Comment dès lors les accompagner au mieux dans ces moments qui questionnement?

Cet article vise à montrer comment par un dispositif de formation-action réflexif une démarche de professionnalisation peut être, d'une part un processus de construction des sujets leur permettant de prendre conscience de leur bagage d'expérience, de leur professionnalité et, d'autre part un processus contribuant à la construction de leur posture d'apprenance, de sujet apprenant. II s'agit d'un processus en mouvement qui s'inscrit dans la philosophie de la formation et l'orientation tout au long de la vie, dans lequel le sujet doit se reconnaître comme un acteur au travail, comme un auteur de son travail et comme un sujet en travail sur son activité constructive favorisant ainsi son développement.

Title: To accompany one moment of professional transition with a reflexive device of formation-action carried out near accompaniment professionals and careers advisers

Keywords: Apprenance - Reflexivity - Oneself - the Council - Professional Transition — Professionalisation - Experiment

Abstract: The question of the professionalisation of the actors often makes debate because it could imply that an actor who professionalises is not a good professional even that he is not qualified. This position is all the more reinforced when these actors live moments of professional transition, where the doubts and the uncertainties come to push aside their professional identity. How consequently as well as possible to accompany them at best in these moments which re-questioning?

This article aims at showing how by a reflexive plan of training-action an approach of professionalisation can be, a process of construction of the subjects which allows them to become aware of their luggage of experiment, their professionality and, on the other hand which contributes to the construction of their posture of apprenance. It is about a process in movement which joins in the philosophy of the training and the orientation throughout the life, in which the subject has to recognize itself as an actor in the work, as an author of its work and as a subject in work on its constructive activity supporting its development thus. 
Cette contribution propose un retour d'expérience sur un dispositif de formation-action réflexif qui s'adresse aux conseillers de l'orientation et de l'accompagnement professionnels dans le cadre de la mise en place du conseil en évolution professionnelle (CEP), une nouvelle prestation de service proposée par la loi sur la formation professionnelle, l'emploi et la démocratie sociale, du 5 mars 2014.

Tout d'abord, il est important de présenter la position de l'auteure. Je travaille depuis plus de quinze ans dans le domaine de l'orientation et de l'accompagnement professionnels des adultes, en tant praticienne et en tant que chercheure impliquée dans la recherche-action. Cette double casquette m'a permis d'être confrontée au réel du travail des conseillers tout en essayant de le rendre intelligible et de me confronter ainsi à la question du sens que ces professionnels attribuent à leur travail. Mon fil conducteur est de chercher à comprendre comment concilier la double dynamique évolutive des compétences et des situations de travail.

Directrice du Centre Cnam Paris aujourd'hui, mes fonctions antérieures de responsable nationale de l'orientation, du conseil et de l'accompagnement des adultes et des organisations au Cnam, m'ont permis de développer des actions de formation et des prestations de services liées aux activités d'AIOA ${ }^{1}$ et, d'accompagner au niveau national des acteurs, internes, mais aussi externes, exerçant dans ces domaines, dans leur démarche de professionnalisation.

Ce conseil en évolution professionnelle (CEP) induit des changements, questionne la posture des conseillers chargés de I'AIOA des cinq opérateurs identifiés par la loi (Apec, Pôle emploi, Fongecif-Opacif, Cap emploi, UNML) et celle de tous ceux qui sont concernés de près ou de loin par ce conseil en évolution professionnelle (consultants, conseillers RH, personnels IOIP des universités, etc.). En effet, ces évolutions amènent à repenser leur cadre de travail, leurs contextes professionnels, mais aussi les activités qu'ils mobilisent et, le sens de leur travail.

Comment dès lors les accompagner dans cette période de transition professionnelle qui s'engage, en sachant que ces évolutions ne sont ni voulues ni souhaitées par les acteurs concernés, mais imposées par un cadre législatif? C'est en ce sens que nous considérons le CEP comme un événement dans la pratique des conseillers.

Pour aider ces professionnels à préparer cette transition, nous, praticiens et chercheurs des équipes «métiers de la formation » et Inetop ${ }^{2}$ du Cnam, avons conçu un dispositif de formation-action basé sur la réflexivité. II s'agira dans ce texte de présenter un retour d'expérience suite à la mise en œuvre de cette formation, suivie à ce jour par plus de cent cinquante conseillers déjà expérimentés dans d'autres formes de conseil en orientation, d'institutions différentes et, qui se préparent à une nouvelle fonction du conseil. Notre matériau recueilli montrera ce que ces conseillers expriment au cours de cette phase initiale de formation avant la découverte du conseil en évolution professionnelle et de l'activité qui y est liée.

Pour structurer notre propos, nous présenterons dans un premier temps le dispositif de formation-action proposé aux conseillers en orientation et en accompagnement professionnels dans le cadre de la mise en place du CEP. Dans un second temps, nous présenterons notre cadre théorique de référence : celui de la didactique professionnelle (Piaget, 1974; Pastré \& Samurcay, 2004; Rabardel, 2004) croisé avec l'approche des transitions professionnelles (Morrison, 1993), Nicholson, 1984; Dubar, 1998; Schlossberg; 2005) et celle de l'expérience formatrice (Jorro, 2011). Dans un troisième temps, nous présenterons les premiers résultats des travaux conduits avec les conseillers ayant suivi la formation, à partir d'un cadre méthodologique qui met en évidence les stratégies de prise de décision de ces acteurs face au CEP, intégrant représentations, valeurs, rôles et mode d'implication professionnelle. Ces résultats apporteront des éclairages sur la manière dont une démarche réflexive peut contribuer à la professionnalité des conseillers de l'accompagnement professionnel, le type de questions transversales que

$1 \quad$ AIOA : Accueil Information Orientation Accompagnement

2 INETOP : Institut national des études sur l'orientation professionnelle 
cela pose sur le plan des compétences professionnelles, des registres de savoirs, des dynamiques et constructions identitaires et comment finalement ces professionnels de l'accompagnement professionnel s'inscrivent dans une posture d'apprenance.

\section{La démarche de construction du dispositif de formation-action réflexif}

\subsection{Une construction basée sur une expérience antérieure}

La construction du dispositif de formation-action mis en place dans le cadre du CEP s'appuie sur une expérience, de nature voisine, conduite depuis 2011, qui a consisté à développer la politique AIOA du Cnam suite à une période de réorganisation de l'établissement et à un contexte législatif (loi 2009) qui donne désormais à chacun un droit à l'orientation tout au long de sa vie (Poulin, 2015).

À partir d'une méthodologie basée sur la construction collaborative et participative, un groupe de travail composé de professionnels AIOA représentatifs de l'ensemble des centres Cnam en région a abouti à l'identification d'un modèle de services communs proposés aux publics en matière d'AIOA, à partir des situations réelles de terrain rapportées par les acteurs : de quoi parle-t-on sous les termes AIOA? Que font les acteurs? Quelles activités mobilisent-ils et comment?

Ce qu'il nous semble important de relever ici, c'est que ce travail collectif a conduit à l'élaboration d'une charte nationale AIOA, qui témoigne de l'engagement de qualité de l'établissement en la matière et qui facilite la lisibilité interne et externe des activités liées à ce champ professionnel. Néanmoins cette formalisation écrite des productions du travail, qui devenait un élément lisible en interne (par des pairs, des collègues, la hiérarchie) et en externe (par des acteurs d'autres institutions), a suscité chez certains conseillers un questionnement sur leurs «compétences » et leur «légitimité professionnelle » pour exercer ces métiers du conseil.

En effet, beaucoup de professionnels qui exercent ces fonctions, ont appris sur le tas et n'ont pas tous une formation dite académique «formelle». Ils mettent en œuvre depuis de nombreuses années ces activités AIOA qui constituent de fait leur cœur de métier et leur quotidien de travail. Ils exercent leur travail avec leurs expériences, leurs compétences incorporées (Leplat, 1991), leurs «trucs » et « astuces» et réussissent. Ils sont par ailleurs reconnus par leur institution et se reconnaissent eux-mêmes comme de bons professionnels. Ces acteurs ont mis en place ce que Piaget (1974) appelle une coordination agie basée sur la réussite de leurs actions.

Et en même temps, cette formalisation (la charte), qui donne à voir leur travail en partie, vient soulever des questionnements d'ordre «identitaire au travail»:

«Est-ce que finalement, depuis toutes ces années, je fais mon travail correctement?» «Est-ce que j'ai vraiment le socle solide de compétences pour exercer ce métier?» «Est-ce que j'ai les bonnes compétences pour le faire?» «Est-ce que je suis autorisé(e) à assumer ces fonctions alors que je n'y suis pas formé(e) formellement?»

S'exprime alors chez ces acteurs un besoin de réassurance, de réaffirmation, de reconnaissance.

Pour apporter des premiers éléments de réponse à ce besoin, nous avons choisi de construire un dispositif de formation action très court ( 3 jours), basé sur les échanges d'expériences et centré sur l'appréhension du contexte professionnel du conseiller AIOA du Cnam.

L'idée centrale est de favoriser une démarche de compréhension chez le professionnel : compréhension de son travail, compréhension de ce qui est attendu de lui par ses pairs, sa hiérarchie, ses collègues et, compréhension de ce que lui-même attend de son travail. Apprendre à se centrer, pas uniquement sur la réussite de son travail, mais sur le «comprendre ce qui se joue» pour lui au travail.

En place depuis septembre 2011, plus de 260 professionnels de I'AIOA ont suivi à ce jour cette formation et confirment qu'elle répond à leur de besoin de réassurance, par les contenus proposés en lien direct avec les terrains professionnels et les études de cas proposées; à leur besoin de réaffirmation qui par le collectif de travail engagé recrée une dynamique de réseau entre collègues; et enfin à leur besoin de reconnaissance, car être au clair sur son contexte professionnel favorise l'identification de son rôle en tant qu'acteur au travail.

L'animation de ce dispositif, que nous avons réajusté, réaménagé au fil des besoins des acteurs de terrain et qui a aujourd'hui une forme «stabilisée», a constitué un réel atout dans la démarche de construction du dispositif de formation dans le cadre du conseil en évolution professionnelle que nous allons maintenant présenter. 


\subsection{Co-construction d'un dispositif de formation-action dans le cadre du CEP}

Un besoin d'accompagnement induit par un texte réglementaire...

En veille sur ces métiers, nous avons été très attentifs à la construction du CEP, nouvelle prestation gratuite, accessible à tout public salarié dès l'âge de 16 ans indépendamment de son statut et de sa qualification (arrêté du 16 juillet 2014 fixant le cahier des charges relatif au conseil en évolution professionnelle prévu à l'article L.61111-6 du code du travail).

Dès les premiers projets de cahier des charges relatifs au CEP, notre attention a été attirée par un point : «la professionnalisation des équipes en charge de l'offre de services du conseil en évolution professionnelle». Notons que c'est la première fois que dans un texte réglementaire la question de l'accompagnement des professionnels, exerçant dans la relation de service et d'aide à autrui, est spécifiée. Ceci pose dès la création du CEP, une hypothèse de changement pour les professionnels, d'adaptation à ce nouveau service, raisons pour lesquelles un accompagnement dans la démarche sera proposé aux cinq opérateurs identifiés. Nous pourrions nous poser la question du pourquoi cette attention particulière sur l'accompagnement des professionnels? Mais là n'est pas notre propos.

\section{Un besoin d'accompagnement exprimé par les acteurs de terrain...}

Le besoin d'accompagnement s'est exprimé d'emblée chez les conseillers de terrain par un besoin de formation facilitant la recherche de la boîte à outils, pour mettre en œuvre le CEP.

Les centres Cnam en région ont été nos antennes au niveau national pour identifier les besoins des acteurs de terrain, car ils sont en relation directe avec des opérateurs potentiels locaux du CEP (missions locales, Pôles emploi, Carif, etc.). Les questions récurrentes recueillies étaient : «comment allons-nous mettre en œuvre ce CEP?»; «il faut que je me forme, ce n'est pas mon métier»; «ce CEP propose trois niveaux, mais c'est complexe!» Des questionnements très proches de ceux des conseillers AIOA du Cnam évoqués précédemment, car ces professionnels ont des profils similaires. D'autres, par exemple comme les conseillers en bilan de compétences, se sont sentis d'emblée menacés dans leur activité, car ils perçoivent le conseil en évolution professionnelle comme une prestation concurrente à celle du bilan de compétences; «le bilan de compétence va disparaître », «le CEP est un bilan de compétence déguisé... », «les bilans de compétences n'auront plus de financement... !», "c'est le pot de terre contre le pot de fer!»

Parallèlement, nous avons mené une veille documentaire et avons eu des échanges de travail multiples avec les acteurs institutionnels qui ont participé à l'écriture du cahier des charges du CEP (DGEFP, rapporteur de la loi, FPSPP, etc.). Là aussi la question de l'accompagnement des conseillers a toujours été au cœur des discussions et une priorité.

Comment dès lors accompagner ces acteurs de terrain dans ce moment de transition professionnelle?

\section{Un dispositif pour accompagner la transition professionnelle...}

Le constat que nous pouvons partager c'est que ce conseil en évolution professionnelle vient réinterroger l'activité de tous les prescripteurs de conseil en orientation et en accompagnement professionnel. Ce travail préparatoire d'analyse des besoins nous a permis de poser notre postulat de départ et de considérer le conseil en évolution professionnelle comme le vecteur d'une période de transition professionnelle pour les conseillers AIOA, qui les conduit à des remaniements identitaires (Poulin, 2015).

Le cadre théorique qui soutient notre démarche et que nous détaillerons plus loin dans ce texte est celui de l'analyse de I'activité par la didactique professionnelle, dont le postulat est de comprendre pour transformer, accompagner. Le moment de transition, que nous appréhenderons à partir de l'approche de Schlossberg (2005), est celui qui questionne la transformation et qui permet de comprendre le sens de cette transformation à partir des stratégies de prise de décision de ces acteurs face au CEP. Pour cette raison, notre démarche de professionnalisation s'inscrit dans une visée réflexive, centrée sur une dynamique de compréhension, intégrant représentations, valeurs, rôles et mode d'implication professionnelle des acteurs concernés. II s'agit de repartir de leur posture professionnelle actuelle pour envisager avec eux leur évolution en termes de sphère professionnelle en tenant compte de leur bagage d'expérience.

Aussi, depuis octobre 2014, nous proposons une formation-action courte sur trois jours, intitulée «Conseil en évolution professionnelle : éléments de prise en main et de positionnement». Les objectifs de cette formation visent à comprendre le CEP en termes réglementaires et législatifs, à obtenir les premières clés de lecture pour appréhender au mieux les contours du CEP 
et à envisager pour chacun les évolutions de sa posture de conseil professionnel par un questionnement de sa pratique. Ce module s'adresse à tout professionnel ayant une expérience du conseil et de l'accompagnement, du secteur public ou privé, souhaitant se professionnaliser sur le conseil en évolution professionnelle.

Par ce travail réflexif, nous amenons les conseillers à construire un dispositif de questionnement et d'auto-positionnement sur les enjeux de la réforme, sur leur pratique professionnelle, et à appréhender le contexte de travail du conseiller en évolution professionnelle. Il ne s'agit pas de former aux métiers du conseil, mais d'accompagner des conseillers déjà en fonction, expérimentés sur une forme de conseil dans un moment de questionnement et de transition professionnelle.

Inscrits dans une conception développementale du sujet, nous faisons l'hypothèse que notre dispositif va engager le conseiller à utiliser la transition pour faire des choix dans un environnement qui se transforme. "Comment je vais saisir les opportunités et les contraintes du contexte pour accompagner ma transition professionnelle?» "Quels sont mes repères antérieurs?» «Comment ajuster mes acquis à la nouvelle donne?»Cette conception sous-entend l'idée que c'est le conseiller qui décide, qui s'engage et qui se met en projet. II est responsable dans ce processus.

\subsection{Un dispositif pour appréhender les activités par la réflexivité Un dispositif qui mise sur la manière d'agir...}

Les travaux de Carré (2012) sur la posture d'apprenance apportent un bel éclairage sur cette capacité à agir inscrite dans une dynamique de formation tout au long de la vie. Ce qui est visé à travers l'exploration de la posture d'apprenance, c'est bien une ouverture de la personne à la nouveauté, à l'exploration de nouveaux horizons. L'hypothèse est de considérer que si un sujet est au clair avec sa posture d'apprenant alors il peut modifier son action en connaissance de cause, en résonnance avec lui-même. Il peut reconfigurer ce qu'il a à faire et peut rendre explicite ce qu'il a pu acquérir dans son bagage d'expérience. Cette prise de conscience passe par un travail d'explicitation sur les acquis constitutifs de son bagage d'expérience qui va bien au-delà d'un ciblage de ses compétences. II s'agit d'un travail sur le «Soi» en contexte et en écho avec des situations dans lesquelles le sujet a grandi.

Ce paradigme place notre observation de chercheur sur le sujet et non pas sur l'action c'est-à-dire sur le sujet dans l'action et non sur l'action que le sujet produit. Nous interrogeons la posture de conseil selon le prisme du conseiller dans l'action, car en mettant en évidence ce que construit le conseiller au travail nous lui donnons accès à son activité constructive, source de son développement au travail.

De plus, travailler sur cette posture d'apprenance, cette manière d'agir c'est travailler sur des situations, des vécus que le sujet a mis en œuvre avec sa manière d'agir. Ardoino (1990) parle de différentes postures; celle d'agent «je suis conforme au cadre», celle d'acteur «je m'adapte au cadre en y dérogeant quelque peu»; et celle d'auteur « et si je faisais autrement que ce que me propose le cadre?». Toute personne est, à des moments de son itinéraire de vie, "agent», "acteur», "auteur», car chacun est en mobilité permanente entre ces différentes postures.

\section{Un accompagnement qui questionne l'expérience des conseillers...}

Dans notre démarche de compréhension, nous avons interrogé la terminologie de «conseil en évolution professionnelle». Pourquoi ce terme d'«évolution professionnelle» et pas un autre terme plus en vogue par exemple «développement professionnel», qui nous semble-t-il, aurait fait plus spontanément appel à une approche par compétences, à une approche plus conformiste de l'acteur, plus en conformité à un référentiel? Un cadre dans lequel néanmoins il aurait été plus difficile de mettre en évidence les «qualités», les «habiletés» professionnelles de l'acteur. Était-ce l'hypothèse sous-entendue par les créateurs de ce CEP qui, tout en structurant le CEP sur trois niveaux dans le cahier des charges, accordent une place importante à la liberté d'action des conseillers concernant sa mise en œuvre? En effet, aucune contrainte en termes de moyens, de durée, d'approches, de méthodologies de travail n'est spécifiée.

En tant que praticiens-chercheurs, souhaitant engager ces professionnels à réfléchir sur leur posture, leurs habiletés au travail, leurs astuces, nous avons appréhendé le CEP avec l'idée que l'«évolution professionnelle», ne voulait pas dire forcément progrès, mais qu'elle renvoie bien à la notion de progression, qui implique une conception développementale du sujet, avec une hypothèse forte selon laquelle toute personne qui agit quotidiennement apprend. Apprendre c'est apprendre quelque chose et quelque chose sur soi, c'est faire «l'épreuve de soi» (Malet, 1998, p.65). 
C'est pourquoi par notre dispositif de formation-action nous amenons les conseillers en orientation et accompagnement professionnels à prendre en compte cette nouveauté - le CEP — à partir de leur bagage expérientiel et non comme un élément extérieur à conquérir à partir de la question «suis-je capable de?». II s'agit là d'un changement de paradigme pour ces professionnels qui ne va pas de soi, car, comme le montreront les résultats, ils appréhendent le CEP d'emblée par la question «suis-je capable de?» et abordent leur besoin de formation sur ce même registre c'est-à-dire par une demande d'outils.

La démarche réflexive engagée questionne le regard porté sur l'expérience. Il y a souvent un regard déficitaire sur l'activité professionnelle, car en prendre conscience ne va pas de soi. Les personnes ont globalement du mal à se valoriser. Même si des dispositifs tels que la $\mathrm{VAE}^{3}$ par exemple ont permis de faire progresser la prise en compte de l'expérience dans les démarches de certification, cette démarche par la réflexivité ne va pas de soi. Elle permet de focaliser sur l'agir en situation c'est-à-dire sur «le comment» un sujet investit une situation professionnelle, personnelle, extra-professionnelle. Elle permet d'explorer et de comprendre à partir de quelle capacité à inventer en contexte ces sujets agissent. Selon quelle manière d'agir? Avec quelle posture de sujet au travail?

C'est par cette démarche réflexive qu'il est possible de dire qu'il existe réellement un «art du quotidien» au travail en lien avec le travail réel laissant place à la créativité et à un certain degré de liberté, pas toujours conscientisés par les sujets. Ils ont du mal à repérer ces situations d'ingéniosité, d'habiletés au travail, car elles sont trop peu sollicitées par leur propre registre de pensée centré davantage sur la réussite de l'action.

L'évolution professionnelle ne serait-elle pas une occasion de remettre des points d'appui en évidence sur le métier de conseil? Le travail du conseiller ne consisterait-il pas à renvoyer un regard autre, une reconnaissance du travail, un regard valorisant à travers lequel son rôle de portage (Vigotsky, 1997) prendrait tout son sens? Cette reconnaissance consiste à rouvrir l'énergie professionnelle, la capacité à être ingénieux, car les sujets sont toujours beaucoup plus «riches» que ce qu'ils en disent.

Pour ces raisons nous considérons que l'expérience réfère au primat de l'action vécue. Le philosophe américain Dewey (1938) considère l'expérience comme la vie même: «elle emprunte aux expériences antérieures et modifie la qualité des expériences ultérieures». Cet auteur souligne ainsi le critère de continuité de l'expérience. Le second critère est le critère d'interaction qui met en évidence l'action vécue dans un contexte particulier. L'expérience permet ainsi la croissance de la personne et participe à l'élargissement de ses connaissances ainsi qu'à son développement.

Pour Schwartz (2010), l'expérience est liée à la temporalité et au processus de sélection auquel est confronté l'acteur qui, de façon continue, expérimente des situations à partir desquelles il construira des connaissances. "L'expérience ne peut être formatrice que si l'on suppose chez cet être une tentative continue d'intégrer les événements...; nul ne sait exactement qui il est quand il fait expérience ni exactement en quoi elle consiste» (Schwartz, 2010, p. 12).

Parmi les trois grandes conceptions de l'expérience, l'expérience passagère, qui ne provoque pas chez l'acteur de modification importante «j'ai fait l'expérience de... »; l'expérience capitalisée qui représente la somme des actions vécues par l'acteur dans des situations d'interactions diversifiées (Tardif \& Lessard, 1999), c'est la conception de l'expérience comme formatrice constituant le chaînon intermédiaire entre l'expérience passagère et l'expérience capitalisée (Jorro, 2011) qui a conforté notre choix du dispositif réflexif pour appréhender les transitions professionnelles et la dynamique de professionnalisation qui les accompagne.

En effet, l'expérience formatrice est éprouvée, car elle mobilise l'acteur au plan corporel, émotif et cognitif. Elle est délimitée en interpellant l'acteur sur une situation, un événement donné. Cette expérience formatrice est réfléchie, au sens où elle fait appel à l'appréciation de l'acteur et attestée, car l'acteur est capable de rendre compte des apports et des limites de cette expérience. Enfin ce qui nous place au cœur de la problématique de l'analyse de l'expérience et des apprentissages expérientiels en situation de travail c'est que cette expérience formatrice est intégrée au capital de connaissances de l'acteur et est disponible pour faire face aux situations à venir (Jorro, 2011).

Cette idée d'expérience formatrice est exigeante, car il faut se centrer sur la qualité de celle-ci. Les points d'appui de l'expérience sont les acquis. Elle nécessite de se poser des questions : «En quoi cette expérience est-elle formatrice pour construire mon horizon professionnel?» Elle projette l'acteur vers une professionnalité émergente.Pour résumer, nous proposons dans le tableau suivant une comparaison entre l'approche des activités par l'outil et l'approche des activités par la réflexivité qui a guidé le processus de construction de notre dispositif de formation-action dans le cadre du CEP.

3 VAE : validation des acquis de l'expérience 
Tableau 1

Comparaison entre la démarche d'analyse des activités par l'outil et la démarche d'analyse des activités par la réflexivité

(Poulin, 2015)

\begin{tabular}{|c|c|c|}
\hline & Analyse des activités par l'outil & Analyse des activités par la réflexivité \\
\hline $\begin{array}{l}\text { Type de ques- } \\
\text { tionnement : }\end{array}$ & $\begin{array}{l}\text { Questionnement centré sur l'objet: } \\
\text { "Qu'est-ce qu'il faut que j'acquière } \\
\text { pour pouvoir réaliser un CEP?» } \\
\text { "Quels sont mes besoins pour faire du } \\
\text { CEP?» }\end{array}$ & $\begin{array}{l}\text { Questionnement centré sur la pratique: } \\
\text { "En quoi et comment le CEP questionne } \\
\text { ma pratique de conseil ?» } \\
\text { "Qu'est-ce que j'ai déjà acquis dans mes } \\
\text { expériences qui pourrait m'aider à réaliser } \\
\text { un CEP?» }\end{array}$ \\
\hline & $\begin{array}{c}\text { Bagage } \\
\text { d'expérience? }\end{array}$ & $\begin{array}{c}\text { Bagage } \\
\text { d'expérience }\end{array}$ \\
\hline Focus & $\begin{array}{l}\text { Focalisation sur ce qui manque et ce } \\
\text { qui reste à acquérir. } \\
\text { Modèle centré sur l'action du praticien. }\end{array}$ & $\begin{array}{l}\text { Focalisation sur ce qui existe, sur l'acquis qui } \\
\text { n'a pas encore été valorisé. } \\
\text { Modèle centré sur le praticien en activité, sur } \\
\text { l'agir en situation. }\end{array}$ \\
\hline Hypothèse : & $\begin{array}{l}\text { L'acquisition de nouvelles compé- } \\
\text { tences va me permettre de... }\end{array}$ & $\begin{array}{l}\text { La relecture de mes expériences acquises, } \\
\text { de mon vécu, va me permettre de... }\end{array}$ \\
\hline $\begin{array}{l}\text { Stratégie d'ac- } \\
\text { tion : }\end{array}$ & $\begin{array}{l}\text { Stratégie procédurale par l'outil, les } \\
\text { compétences et les référentiels pour } \\
\text { construire sa «boîte à outils» } \\
\text { Objectif : réussir (coordination agie) }\end{array}$ & $\begin{array}{l}\text { Stratégie analytique par le questionnement } \\
\text { de l'expérience du praticien pour construire } \\
\text { sa pratique du CEP } \\
\text { Objectif : comprendre (coordination } \\
\text { conceptuelle) }\end{array}$ \\
\hline $\begin{array}{l}\text { Type d'appre- } \\
\text { nants en situa- } \\
\text { tion }\end{array}$ & $\begin{array}{l}\text { Agents «je suis conforme au cadre» } \\
\text { Acteurs «je m'adapte au cadre en y } \\
\text { dérogeant quelque peu» }\end{array}$ & $\begin{array}{l}\text { Acteurs «je m'adapte au cadre en y déro- } \\
\text { geant quelque peu» } \\
\text { Auteurs «et si je faisais autrement que ce } \\
\text { que me propose le cadre?» }\end{array}$ \\
\hline Temporalité & Courte & Longue (maturation - appropriation) \\
\hline Expérience & $\begin{array}{l}\text { Activité productive (ce qui émane de } \\
\text { l'action directement) : répondre à une } \\
\text { question } \\
\text { Expérience passagère et capitalisée }\end{array}$ & $\begin{array}{l}\text { Activité constructive (ce que construit le su- } \\
\text { jet au travail) : mettre en questionnement } \\
\text { Expérience formatrice }\end{array}$ \\
\hline $\begin{array}{l}\text { Observation du } \\
\text { chercheur }\end{array}$ & L'action que le sujet produit & Le sujet dans l'action \\
\hline
\end{tabular}

\section{Cadre théorique de notre approche}

Le moment de transition professionnelle engagé par le conseil en évolution professionnelle (CEP) pose la question de la compétence des conseillers accompagnateurs qui revient toujours très vite sur les devants de la scène dans ces moments de changement et de questionnement.

La didactique professionnelle...

Notre choix s'est tourné vers la didactique professionnelle, car elle propose un cadre pour appréhender la notion de com- 
pétences, à travers la conceptualisation dans l'action professionnelle, ce qui implique nécessairement une recentration sur l'acteur en tant que sujet pensant, intentionnel et agissant au travail.

En effet, la phase de transition et d'appropriation de cette nouvelle situation de conseil - le CEP — propose une double dynamique:

- $\quad$ une dynamique des compétences liées à l'appropriation progressive par les acteurs de ce CEP et au développement de nouvelles formes de réalisation du travail;

- $\quad$ et une dynamique de la situation de travail de ces acteurs qui va concerner l'organisation du travail et de la production.

- Pour comprendre cette double dynamique, la didactique professionnelle porte son attention sur deux points :

- $\quad$ examiner l'apprentissage du point de vue de l'activité, avec cette idée qu'apprendre, dans le champ des activités professionnelles, ce n'est pas d'abord assimiler des savoirs, mais c'est apprendre à agir de façon durablement efficace. Les savoirs professionnels, techniques ou scientifiques deviennent un élément parmi d'autres qui permet d'organiser l'activité.

- Regarder l'apprentissage du point de vue du développement du sujet, plus précisément du point de vue du développement de ses compétences. On ne se concentre pas sur la situation de travail, mais sur la manière dont l'opérateur se l'approprie et s'y adapte. On s'intéresse à l'acteur dans son devenir, à la dynamique de développement de ses compétences. La didactique professionnelle va analyser les conditions et les processus par lesquels se construisent, se déconstruisent et se reconstruisent les compétences professionnelles d'un professionnel.

Dans cet article, nous n'aborderons pas l'ensemble du mouvement de «construction — déconstruction — reconstruction » en situation de travail. Nous l'appréhenderons par les représentations que les acteurs se font de ce mouvement, alors qu'ils se situent encore dans une phase de découverte du CEP.

La transformation du contexte de travail implique une évolution des compétences et provoque chez les acteurs un sentiment d'évolution des compétences. Dans le contexte étudié d'arrivée du CEP, on voit comment certains conseillers se sentent prêts à envisager de réinvestir leurs compétences acquises, dans d'autres situations de conseil, dans la situation future du CEP et comment d'autres ne s'y sentent pas du tout prêts.

En effet, le CEP met les conseillers avec lesquels nous avons travaillé dans une attitude proactive qui nécessite une anticipation des tâches et de l'action à venir, qui conduit à rechercher des informations concernant la manière de réaliser les tâches mentionnées dans le cahier des charges du CEP (arrêté du 16 juillet 2014). Tâches et formes d'action qui n'ont pas encore été rencontrées effectivement, car le CEP n'a pas encore été mis en œuvre par les acteurs rencontrés. Nous pouvons dire que cette anticipation se rapproche ici des caractéristiques de la planification de l'action : «[...] la conception anticipée d'une procédure d'action, sans accès à la rétroaction apportée par l'exécution effective» (Hoc, 1990). Le conseiller anticipe la situation future sans connaître les indices qui lui seront fournis par la situation future CEP.

Cette posture d'anticipation questionne les conseillers, car elle les interpelle sur la compréhension de l'action alors qu'ils sont centrés davantage sur la réussite de l'action à réaliser, sur «le faire». Une centration qui les réconforte, car elle leur permet de se mettre en regard des contenus attendus du cahier des charges du CEP «Que dit le cahier des charges? Quelle structuration par niveau? Quels objectifs de sortie pour les différents niveaux du CEP?» : Une approche par l'outil (cf. tableau p.10). Notre rôle consiste à les interpeller sur la compréhension de l'action et les questions que nous leur posons immédiatement après, sont : «Vous, qu'en comprenez-vous? Quelle représentation vous en faites-vous de votre fenêtre actuelle de conseiller?»

Piaget (1974) montre que dans bon nombre de situations liées à l'action humaine, il existe un écart entre la réussite de l'action et la compréhension de cette réussite par le sujet. Dans des activités où un sujet atteint très vite la réussite pratique, il y a un décalage temporel important entre réussir et comprendre, donc entre la coordination agie et la coordination conceptuelle (Pastré, 1997).

La posture d'anticipation peut convenir au conseiller qui est plutôt dans une posture d'«acteur» voire d'«auteur» de sa stratégie d'action, qui a déjà engagé des phases de compréhension de son activité au sens de la coordination conceptuelle de Piaget. Mais pour le conseiller qui est davantage dans une posture d'«agent» (Ardoino, 1990), centré sur la réussite de I'action, la situation d'anticipation est déstabilisante, provocatrice de stress, car sa mise en œuvre, son cadre «pour agir» reste à construire. 


\section{Les transitions professionnelles...}

L'autre élément théorique que nous avons convoqué, qui favorise également la centration sur le sujet pensant, intentionnel et agissant dans sa dynamique de construction professionnelle concerne les transitions professionnelles. "Tout évènement ou absence [d'un évènement prévu] qui a pour effet de transformer les relations, les routines, les croyances et les rôles [de l'individu]» (Schlossberg, 2005, p. 27). Le conseil en évolution professionnelle est un de ces événements non prévus qui vient interroger les relations de travail du conseiller.

C'est aussi pour cette raison que nous envisageons la transition comme un «espace-temps de co-construction du changement individuel et social dans lequel chaque trajectoire socio - professionnelle est liée à la fois à un contexte socio-économique, culturel et aux stratégies singulières intégrant représentations, valeurs, rôles, modes d'implication professionnelle» (Balleux et Perez-Roux, 2013). Un espace-temps de passage inscrit au cœur d'un changement, assumé ou non, et qui nécessite de l'individu la mise en œuvre de stratégies d'adaptation pour mieux gérer éléments de rupture et (re) construction de continuités (A. Balleux \& T. Perez-Roux, 2013, p.102). Aussi la transition induit l'idée d'un passage où se jouent des processus et des stratégies d'adaptation (Tapia, 2001), l'idée d'une «zone intermédiaire d'expérience» (Boutinet, 2009, p. 207).

En clair, il faut du temps pour gérer une transition professionnelle et cette dimension temporelle de la dynamique de compétences en situation de travail est aussi importante. L'empan temporel est essentiel, car l'activité constructive du sujet en situation de travail ne peut être saisie que sur du moyen voire du long terme.

Deux approches de la transition sont possibles et ne couvrent pas les mêmes temporalités (Balleux \& Perez-Roux, 2013). La première tente de borner de façon objective un temps donné, extérieur au sujet (recherche d'emploi, début de formation professionnelle, etc.); la seconde reste liée aux processus vécus par les sujets et aux remaniements identitaires inscrits dans des durées variables selon les individus et les contextes. C'est dans cette seconde approche que s'inscrit la construction de notre dispositif réflexif.

La transition est dès lors envisagée comme une chance de développement pour l'individu, de son parcours ou comme un moyen de retrouver, de renforcer ses valeurs personnelles et professionnelles et d'engager un travail sur soi, de réflexivité qui lui serve de levier dans sa pratique.

Les phases de la transition requestionnent les manières de produire, de penser et d'agir des états, des groupes et des individus.

Erikson a été l'un des premiers à considérer les transitions comme des moments clés du développement et à les envisager comme faisant partie d'un tout existentiel (Cohen-Scali \& Guichard, 2008). La transition est alors indissociable du développement humain et est un passage inévitable dans la construction de l'identité.

$\mathrm{Si}$ «les transitions professionnelles modifient les rôles, les relations et les façons de penser» (Schlossberg, 2005, p.85), elles sont variables en fonction des caractéristiques de l'individu (sa situation, ses ressources, ses stratégies, sa force de mobilisation), de l'environnement et des richesses sur lesquelles il peut s'appuyer ou pas. Les transitions vécues par les adultes ne sont pas forcément désirées et sont vécues différemment si elles sont anticipées ou non. Les événements eux-mêmes peuvent provoquer des transitions auxquelles les adultes n'ont pas été préparés et aux auxquelles ils sont conviés de leur plein gré ou non (PerezRoux \& Balleux, 2012).

Nous abordons les transitions professionnelles à partir du modèle théorique de Schlossberg (2005) en considérant l'arrivée du conseil en évolution professionnelle comme un vecteur qui occasionne un moment de transition professionnelle non anticipé et non attendu. Nous définissons les transitions non anticipées comme des événements souvent perturbants qui arrivent de façon inattendue, tels qu'une opération chirurgicale grave, un accident de voiture sérieux ou une maladie, mais aussi une promotion surprise, la fermeture de l'entreprise ou dans le cas présent une nouvelle donne professionnelle, le CEP. Cet événement non attendu est caractéristique de la période transition que vivent les conseillers en orientation et accompagnement professionnels aujourd'hui.

De plus, la transition professionnelle repose sur la dynamique de socialisation professionnelle et Morrison (1993) avec sa théorie de la «proactivité » distingue quatre types d'action qui peuvent aider l'individu dans cette période :

- $\quad$ acquérir une maîtrise suffisante de la tâche professionnelle pour savoir comment remplir efficacement sa fonction:

- clarifier son rôle pour savoir quel comportement ses pairs et son institution attendent de lui;

- s'acculturer pour s'approprier la culture professionnelle; 
- s'intégrer socialement pour connaître les comportements et attitudes jugés appropriés ou non par son groupe et ses collaborateurs.

Par ailleurs Morrison distingue deux canaux permettant de rechercher ces informations qui diffèrent de par leur mise en œuvre, mais aussi par la qualité et le «coût psychologique» qu'ils entraînent :

- la recherche explicite d'information par questionnement direct qui s'opère surtout pour les informations techniques. Dans nos travaux, la situation de formation facilite cette recherche et nous avons structuré ce questionnement sur le CEP en demandant aux conseillers de formaliser leur définition du CEP à partir de la représentation qu'ils en avaient.

- le deuxième canal : l'observation des comportements d'autrui est une manière pertinente pour obtenir des indices et obtenir ainsi d'autres types d'information. Le groupe est un bon support pour faciliter cette observation des pairs. Nous avons engagé ce canal chez les conseillers en leur demandant de coconstruire une définition du CEP avec un autre participant de la formation.

Puis, dans la gestion de cette transition professionnelle, l'individu va chercher à s'approprier une démarche pour repérer des indicateurs afin de favoriser son adaptation professionnelle. Nicholson (1984) montre combien l'individu va avoir besoin de s'adapter en fonction de l'interaction «soi - organisation» ou dit autrement «acteur-contexte». Une dynamique qui va le conduire soit à un changement et un développement personnel, soit à un changement de la situation qu'il vit, soit à opérer les deux à la fois (un changement personnel et un changement de la situation). II est alors interpellé sur son système de valeurs, ses codes de référence, ses attentes, son identité professionnelle.

En alliant les concepts de la didactique professionnelle et de la transition professionnelle évoqués, nous nous centrons sur le «comprendre» ce qui se joue pour les conseillers et nous mettons en perspective une manière dont le conseil en évolution professionnelle est appréhendé par les conseillers.

Nous avons rapproché ces deux courants théoriques, car nous pensons qu'ils posent des principes communs :

Le premier principe est que développement des compétences et transition professionnelle transforment les rôles, les relations, les habitudes et les façons de penser des sujets. Ce n'est pas la transition ou la compétence en soi qui est cruciale, mais le changement que cette transition, cette évolution en compétence induit dans les rôles, les relations, les habitudes et les façons de voir. Comment le conseiller prend-il conscience de la transformation et comment développe-t-il ses ressources? Comment est-il interrogé sur ses mécanismes à l'œuvre dans l'apprentissage à la fois des dimensions «opératoires» (gestes, façons de faire nouvelles) et des dimensions identitaires et culturelles (façons de penser et de concevoir le travail et ses contextes)? Quelle mise en mouvement cela provoque-t-il chez lui? Que comprend-il de l'activité qu'il mobilise? Quelles stratégies analytiques met-il en œuvre? Quel sens donne-t-il à ce changement provoqué par la transition?

Second principe : transition professionnelle et développement de compétences sont inscrits dans une temporalité. Le processus des transitions est long et les réactions des individus changent tout au long du processus. Dans un premier temps, les individus sont submergés positivement ou négativement par leur nouveau rôle. Progressivement, ils commencent à prendre des distances par rapport au passé et à envisager leur nouveau rôle, dans un moment de balancement entre les deux. Le processus d'abandon d'un ensemble de rôles, de relations, d'habitudes et de façons de penser, et d'investissement d'un nouvel ensemble prend du temps. Pour certains, ce processus est facile et rapide, pour d'autres, il peut prendre des années.

Troisième principe : transition professionnelle et développement de compétences engagent les personnes à prendre des décisions et donc à mettre en œuvre des stratégies d'action. En quoi les individus diffèrent-ils dans leur manière d'affronter ce qui pourtant semble une même transition professionnelle? Comment alors traverser cette épreuve? Comment la vivre et comment apprendre de cette expérience?

\section{Cadre méthodologique}

\subsection{Choix épistémologique}

Pour soutenir cette dynamique de compréhension de l'action par les conseillers, notre choix épistémologique a consisté à interroger l'activité des praticiens à partir de leur posture professionnelle. Dans le cadre des modules de formation proposés, nous avons souhaité amener les conseillers à réfléchir sur leurs stratégies analytiques en cherchant à comprendre le «comment» de leurs actions mobilisées, en faisant un autodiagnostic guidé méthodologiquement, c'est-à-dire une évaluation de la situation de conseil dans son ensemble. 
Pour aider les conseillers à réfléchir sur leur propre stratégie pour affronter cette transition non voulue, nous avons travaillé à partir des travaux de Schlossberg (2005) qui permettent d'identifier les caractéristiques des transitions en se centrant sur les changements provoqués et de mettre en évidence les ressources et les insuffisances avec lesquelles chacun affronte les transitions. Cette auteure classe ces caractéristiques en quatre catégories essentielles appelées le Système des 4 S : Situation, Soi, Soutiens, Stratégies.

- La Situation désigne la situation de la personne au moment de la transition. Vit-elle ou a-t-elle vécu d'autres situations de stress?

- Le Soi renvoie à la force personnelle intérieure d'une personne face à une transition. Est-ce une personne optimiste, résiliente, capable d'affronter l'incertitude? Ce que l'on peut mobiliser de soi-même influence la façon dont on fait face à une situation.

- Le(s) Soutien(s) que l'on reçoit ou que l'on peut attendre lors d'une transition s'avère(nt) crucial (ux) pour le sentiment de bien-être.

- Les Stratégies : il n'y a pas de stratégie d'adaptation magique néanmoins une personne qui utilise plusieurs stratégies avec souplesse sera plus capable qu'une autre de faire face. Ce développement de stratégie est en lien avec le vécu de la situation, l'énergie déployée par le sujet et les ressources qu'elle peut mobiliser. Les stratégies sont une mise en mouvement «vers un espace de possibles». Anticiper dans le futur, la résolution d'une problématique, des questionnements, des doutes du présent.

À partir de ces principes, nous avons mis en place un outil réflexif à partir d'une grille co-élaborée avec des conseillers de I'Inetop (Institut national d'études sur le travail et l'orientation professionnelle) au Cnam.

Cet outil réflexif convoque trois éléments :

- I'expérience (construction du sens) : à partir du vécu des conseillers, comment le CEP est-il appréhendé?

- L'apprenance (posture) : en tant que sujet, qu'est-ce que je comprends du CEP? Comment j'envisage de me l'approprier?

- La transition professionnelle (changement) : quelle stratégie d'action j'envisage de mettre en œuvre pour appréhender cette nouvelle donne?

Ces trois éléments «expérience-apprenance-transition» facilitent de notre point de vue le mouvement de construction déconstruction - reconstruction des compétences professionnelles des conseillers, proposé par le cadre de la didactique professionnelle évoqué plus haut. En effet, les conseillers dans leur réflexion convoquent leurs acquis, leur posture pour construire un plan d'action. Leurs acquis d'expérience et leurs compétences constituent leurs ressources propres pour construire leur compréhension du CEP; ce sont les leviers dont ils disposent dans l'immédiat pour développer leur activité productive. Cependant il est important de rappeler que l'expérience a un double statut au sens où elle est le produit de l'activité productive du professionnel et au sens où elle constitue un matériau, un objet travaillé par l'activité constructive de celui-ci. Pour engager le travail sur ce deuxième aspect, les conseillers ont été interpellés dans leur posture en tant que sujets pensants, intentionnels et agissants. Ils sont amenés à déconstruire, à remettre à plat leurs acquis, en se mettant en posture réflexive et en analysant la nouvelle situation pour la comprendre et l'apprivoiser. Suis-je dans une attitude facilitant ma dynamique d'apprentissage dans la situation?

Une fois ce mouvement de déconstruction réalisé, les conseillers ont alors des premiers éléments pour se projeter dans une stratégie pour envisager au mieux pour eux le CEP. Notre hypothèse, c'est qu'à travers ce temps très court ( 3 jours) de formation-action, les conseillers ont été interpellés sur leur posture réflexive, qu'ils ne convoquent pas d'emblée pour certains, et que les prémisses de questionnements engagées leur permettront de chercher et de donner du sens à ce changement professionnel.

Pour terminer, sur cette présentation du cadre méthodologique, il faut préciser que cet outil réflexif a été accompagné par des phases d'écriture (très courtes et régulières), sous-tendues par le paradigme de la réflexivité, pour engager le conseiller à porter un regard rétrospectif sur sa pratique, et mettre en mots une projection vers une nouvelle pratique le CEP : d'action elle devient énoncé sur l'action. Ce nouvel énoncé s'accompagne d'une prise de conscience par le conseiller de modalités d'action auparavant non questionnées qui enrichissent donc ses acquis d'expérience. La formalisation écrite est une occasion d'un apprentissage sur soi. Le fait de réinterroger en formation l'action professionnelle mise en œuvre in situ permet de changer le statut de l'action pour son auteur : ainsi devient-elle pour lui un acquis «su», conscientisé de l'expérience, alors même que l'action considérée pouvait être fortement incorporée à la situation et donc non identifiée par lui. Les différentes 
phases de mise en mots permettent un apprentissage non seulement par le conseiller —auteur de celle-ci —, mais également par les participants partageant en formation le même espace-temps. Les uns apprennent de l'écoute des autres.

\subsection{Présentation des résultats}

Les conseillers formés, plus de cent quatre-vingts à ce jour (sur Paris et en Aquitaine), viennent d'institutions différentes (Pôle emploi, Cité des métiers, universités, Fongecif et Opacif, cabinets de conseil...) et occupent une fonction dans le champ professionnel de I'AIOA (conseiller en orientation, en insertion professionnelle, en formation, chargée d'accueil et d'information...). Ils sont concernés par le CEP directement (pour les acteurs des institutions désignées par la loi) ou indirectement (pour les acteurs qui pourraient potentiellement être désignés par les Régions pour dispenser du CEP).

Ces sessions nous ont permis de recueillir des éléments sur la manière dont ces conseillers appréhendent le CEP, sur le niveau d'information qu'ils en ont et comment ils envisagent leurs situations professionnelles avec cette nouvelle donne. Les résultats seront présentés plus loin.

Notre corpus porte sur un échantillon de 65 conseillers, car les traces des travaux nous ont été confiées sur la base du volontariat et de manière anonyme, pour en effectuer une analyse. Néanmoins les résultats ont été présentés de manière anonyme, sous la forme orale avec un support PowerPoint, à l'ensemble des conseillers de chacun des groupes, qui se retrouvaient tout à fait dans les éléments d'analyse exposés.

\subsubsection{Définitions individuelles du CEP}

Pour avoir quelques éléments sur la démarche proactive des conseillers dans ce moment de transition professionnelle, nous les avons sollicités sur leur niveau d'information sur le CEP. Nous leur avons demandé dans un premier temps de formaliser leur définition du CEP à partir de la représentation qu'ils en avaient.

Exemples de définitions individuelles du «CEP» (du corpus étudié) :

- C'est accompagner un individu qui souhaite un changement professionnel. C'est prendre le temps d'analyser son projet afin de définir ensemble un parcours incluant les actions et orientations à mettre en œuvre pour atteindre son objectif. (A1)

- Le CEP a pour objectif d'accompagner un bénéficiaire dans sa mobilité professionnelle. L'accompagnement consiste à amener le bénéficiaire à construire un projet professionnel réaliste et réalisable jusqu'à sa mise en œuvre. (B2)

- Il s'agit d'accompagner un individu afin d'identifier ses besoins dans le but de réaliser son projet en tenant compte de son parcours. (C3)

- Le CEP permet à un individu d'exprimer un besoin face à un professionnel afin de sécuriser son parcours. Ce qui lui permettra de s'adapter au marché de l'emploi ou de se donner les moyens d'évoluer. (D4)

- Il s'agit d'une rencontre, celle d'un consultant exprimant un besoin à travers sa demande, avec un professionnel sous forme d'un contrat moral afin de pouvoir le guider dans la mise en pratique de la finalité qu'il s'est fixée. Cet échange lui permettra d'assurer son changement, de le faire accompagner dans la durée. Le suivi sera assuré par un référent unique, responsable de la mise en œuvre du projet. Des outils devront aider à la décision et notamment la connaissance de l'emploi et des métiers à travers des territoires. Ces échanges devront être formalisés à travers un document de synthèse pour permettre au demandeur "de tendre à" la réalisation (finalité) des différentes étapes. (E5)

- $\quad$ Permettre à une personne de faire le point sur son parcours en instaurant un échange. Écouter, analyser la demande pour établir un diagnostic. Par la reformulation, se mettre d'accord avec la personne sur la nature et le contenu du cadre du conseil apporté/souhaité. À titre d'exemple : identifier les compétences, mettre en relief les éléments significatifs du parcours. Aider la personne dans son cheminement, à prendre conscience d'éventuels freins/atouts et des adaptations nécessaires au développement personnel et professionnel de la personne. (F6)

- Le conseiller en évolution professionnelle écoute les attentes, identifie les besoins du demandeur. II mobilise ses ressources, propose une méthodologie permettant le choix d'un métier sur un marché de l'emploi défini et aide à la mise en œuvre du projet, selon le cas, en identifiant et en mobilisant les financements possibles, le tout d'un commun accord. (G7)

Ces corpus montrent que certains conseillers envisagent le conseil en évolution professionnelle par l'action et d'autres par ce que cela va induire pour eux-mêmes. 


\subsubsection{Définitions partagées du CEP}

Dans un second temps, pour recueillir quelques éléments sur la dynamique de socialisation professionnelle avec des pairs, nous avons demandé aux conseillers de se mettre par binôme de leur choix et de coproduire une définition du CEP, à partir de leurs représentations partagées.

Exemples de définitions coproduites du «CEP»(du corpus étudié):

- Un dispositif permettant à chacun d'être accompagné dans sa réflexion professionnelle, quel que soit son questionnement. Cette démarche peut impliquer un diagnostic partagé, la formulation d'étapes de travail, la mise en œuvre d'actions afin de poser les bases d'un projet et les moyens d'y parvenir. C.EP.A

- Le conseil en évolution professionnelle est une forme d'accompagnement d'une personne en recherche de sens sur sa vie professionnelle, à travers des rencontres et échanges avec un professionnel de l'orientation avec qui il fixera des objectifs à atteindre, en rapport avec ses attentes, mais aussi la réalité, selon le moment de vie dans lequel il est. C.EP.C

- Une fois, ce projet professionnel défini, cette évolution peut être la reconnaissance des compétences de la personne par elle-même et par son employeur, suivie par exemple d'une action de formation, d'une mobilité interne ou externe, ou d'une reconversion... C.EP.B

- Le CEP permet de mettre à disposition un service en vue d'accompagner une transition professionnelle souhaitée ou non. Dans cette situation, le professionnel du conseil, sur un temps déterminé, aura pour objectif de permettre à l'usager d'aller de l'avant dans son projet professionnel et personnel. Il développera une posture de conseil avec une écoute active qui lui permettra la reformulation des propos de l'usager, et ce, en vue de l'aider à la prise de décision quant à ses questionnements. Des connaissances en ingénierie pédagogique et financière et une maîtrise de l'environnement socio-économique seront nécessaires à la bonne réalisation du conseil. Le temps de rencontre donnera lieu à la production d'un plan d'action. C.EP.D

- C'est un processus réglementaire d'accompagnement d'adulte dans le cadre d'une démarche d'exploration, d'identification et de réalisation d'un projet d'évolution professionnelle tout au long de la vie dont il sera I'acteur. C.EP.E

- Le CEP propose une méthodologie de co-construction du parcours suivant le souhait d'évolution professionnelle de la personne en lui apportant les ressources (acteurs, lois, infos sur le marché de l'emploi, dispositifs tels que le CPF... C.EP.F

Nommer ce qui dérange et partager avec d'autres qui sont dans la même situation peut alors aider grandement et faciliter la compréhension du sujet. Cet échantillon montre que le CEP est considéré davantage par le dispositif (l'outil) et moins par les acteurs qui mettent en œuvre une situation professionnelle. Comme si la coproduction faisait revenir spontanément à la référence, au référentiel, à la normalité.

\subsubsection{La stratégie d'action envisagée par les conseillers}

Pour réfléchir à leur propre stratégie, chaque participant a une grille (format A3) et répond individuellement aux questions par écrit. Cette grille méthodologique basée sur les 4 S (situation — soi — soutien — stratégie) constitue une base pour aider les conseillers à écouter et à agir avec une nouvelle sensibilité.

Comment se mettre en œuvre sur ce CEP? Avec quelles ressources? Avec quels leviers pour acquérir de nouvelles ressources? Comment adapter le réel d'activité à ce nouveau contexte? Comment mobiliser son pouvoir d'agir?

Pour respecter l'anonymat des conseillers, les résultats sont présentés toute institution confondue, en suivant le cheminement de la grille méthodologique des $4 \mathrm{~S}$ (situation — self — soutien — stratégie).

\begin{tabular}{|l|l|}
\hline 1.Situation & 2. Soi \\
\hline 3. Soutien & 4. Stratégie \\
\hline
\end{tabular}

Situation: À travers la situation il s'agit de considérer des facteurs tels que l'événement déclencheur de la transition (ici le CEP), le moment de la transition, son vécu, ses réactions, les changements de rôles sociaux induits, la temporalité et les expériences antérieures de transitions semblables. 


\section{Les questions proposées pour appréhender la situation sont les suivantes :}

Comment est perçue l'arrivée du CEP dans votre pratique? Quel(s) changement(s) dans vos rôles actuels? A quelle(s) autre(s) transition(s) de semblable nature avez-vous été confronté-e? Comment cela s'est-il passé pour vous?

\section{Les changements que le CEP est susceptible d'impliquer dans les rôles actuels...}

"C'est une occasion de faire évoluer les pratiques, mon poste....; «cela va peut-être permettre de clarifier l'activité... ; «il faudrait changer de manager...»; «les missions de chacun vont évoluer, mais comment?» «Nos hiérarchies vont-elles prendre en compte cette nouvelle mission et mettre en place l'organisation adéquate?» «ll faudrait que l'on soit armé en ingénierie financière», «il faut avoir les techniques d'entretien!.... »; «il nous faudrait un accompagnement vers ce nouveau métier»; «il faudrait revoir l'organisation du travail qui existe actuellement dans l'institution»; «il nous sera demandé d'être plus dans l'expertise et moins dans la gestion des flux»; «je suis loin de mon métier initial»; «avoir la responsabilité de valider le projet de la personne reçue! je ne m'y projette pas du tout?... »; «il faudra avoir une plus grande capacité d'écoute... »; «je suis en attente de changement depuis un certain nombre d'années, donc je suis plutôt contente...»; «on n'a pas de cadre, c'est ce flou qui est perturbant»; «on est en transition, alors qu'on accompagne des personnes en transition!»

\section{Les transitions vécues de semblable nature à celle du CEP...}

«Cette transition me fait penser à des périodes de repositionnements professionnels que j'ai eu dans ma carrière »; "à une mobilité professionnelle il y a 10 ans»; "Cela ressemble à un changement de métier connu il y trois ans»; «je n'ai pas eu de vécu semblable »; "ça me fait penser à ma promotion interne » ; “c'est suite à un changement de métier que je suis aujourd'hui dans le domaine du conseil en accompagnement professionnel»

\section{Comment se sont passées ces périodes de transition vécues...}

«J'ai toujours pris mes changements professionnels comme des moments pour faire un effort de compréhension»; «mon évolution de poste m'a permis une «ouverture sur l'entreprise»; «ce moment a été une valorisation personnelle pour moi»; «j'ai confiance en moi, en période de changement je me fis à mon instinct pour m'adapter»; «je suis plutôt de nature optimiste et volontaire au changement»; "dans les changements que j'ai connus, j'ai toujours été bien accompagné, donc ça s'est bien passé »; "un vécu sous contrainte avec l'épée de Damoclès sur la tête»; "mon changement d'institution a été un risque d'un retour en arrière »; " après une période de flottement, le changement a été un plaisir»; "ces moments sont mangeurs en ressources, il faut avoir une bonne capacité d'adaptation, de composition avec les autres collègues»; «mon dernier changement m'a ouvert une perspective, une nouvelle mission, c'est positif».

Ces résultats sur l'auto-évaluation de la situation des conseillers montrent la manière dont les conseillers appréhendent le développement de leurs compétences : ils sont dans un rapport de filiation aux compétences antérieures par continuité ou par rupture. Certains conseillers s'appuient sur des situations qui leur semblent être de même nature, sur du déjà vu, du déjà vécu, pour appréhender le CEP «J'ai déjà vécu des situations similaires, et j'ai réussi à m'en sortir...». Ils sont tentés de transposer leur savoir-faire dans la nouvelle situation en restant dans la filiation des compétences précédentes.

D'autres conseillers au contraire vont opter pour une filiation par rupture, car le CEP est pour eux une situation nouvelle, étrangère, de caractéristiques différentes, qui pose la question du nouveau métier, d'une nouvelle identité professionnelle à construire, et qui suscite chez eux le sentiment du développement d'une nouvelle expertise "C'est un nouveau métier qui va me demander beaucoup d'énergie, c'est super positif!».

Ces résultats confirment bien l'idée qu'il existe une articulation entre les compétences et les situations de travail : les nécessités d'adéquation des compétences aux situations de travail conduisent à une détermination relative des compétences par les situations dans le premier modèle (filiation par continuité). Tandis que l'articulation «compétences - situations de travail » est posée en relation avec des classes de situations de différents niveaux dans le second modèle (filiation par rupture). Le CEP est une nouvelle classe de situation dans les situations de travail des conseillers.

Soi : Le soi prend en compte des caractéristiques personnelles et sociales de l'individu, ses ressources psychologiques (développement de soi, perspectives), son sentiment d'efficacité personnelle.

Les questions proposées pour appréhender le soi sont les suivantes:

Quel est votre sentiment d'efficacité personnelle face à ce nouveau dispositif? Vous sentez-vous prêt à y faire face? A quel moment de votre vie professionnelle ce CEP tombe-t-il? Comment le vivez-vous? 


\section{Le sentiment d'efficacité personnelle des conseillers pour faire face au CEP...}

«Je manque de connaissances actuellement face à ce CEP»; « des désajustements dans mon métier sont à faire»; «le niveau 1 du CEP : c'est bon, sauf concernant les bassins d'emplois; le niveau 2 du CEP : je peux faire un peu, quant au niveau 3 du CEP il est inexistant dans mon périmètre professionnel»; «je me sens capable de proposer du conseil en évolution professionnelle, car je suis persuadée en faire déjà»; «si d'autres y arrivent pourquoi pas moi?»; «avec un sentiment d'efficacité personnel positif»; «je crois en mes capacités»; «j'ai une totale absence de visibilité sur les partenaires»; «il faudrait que j'aie plus de moyens »; «il faudrait que j'aie des compléments sur la construction des parcours, sur les conditions de financement».

\section{Dans quelle mesure vous sentez-vous prêt(e) à y faire face?}

«Je compte sur mes expériences»; «Je connais l'accompagnement»; «Je connais déjà les publics»; «J'ai déjà accompagné les publics envisagés»; «Je suis entièrement prêt»; «J'aviserai en temps voulu».

\section{A quel moment de votre vie professionnelle le CEP tombe-t-il ?}

"Ce CEP arrive à une année charnière pour moi»; «il arrive à ma deuxième partie de carrière»; «je suis en fin de parcours professionnel»; «Il arrive à un moment de transition »; «il arrive à un moment où j'ai besoin de changement après 7 ans sur le même poste»; «il arrive à une période stable de mon parcours»; «à aucun moment particulier»; «ma vie professionnelle est plutôt stable».

\section{Comment est vécu ce moment de transition professionnelle? Avec quelles perspectives?}

"Je le vis bien»; «une perspective d'évolution de mes compétences avec peut-être un changement de fonction »; "J'espère avoir de nouveaux horizons professionnels»; «c'est une opportunité d'évolution de mon poste qui commence à devenir routinier»; «ce moment est comme un challenge pour moi»; «je suis impatiente que les choses se mettent en place... »; «je suis actif et volontaire, mais en sachant que le risque existe»; "cela dépendra de l'organisation mise en œuvre et de mes futures missions»; «j'ai envie d'apprendre, mais pas envie de faire n'importe quoi».

Les résultats relatifs à l'auto-évaluation du soi des conseillers montrent que ce moment de transition est vécu avec des perspectives positives en termes de projet et d'évolution professionnelle, de renouvellement d'activité. Les conseillers expriment un sentiment d'efficacité personnelle plutôt positif. Ils se sentent prêts pour faire du CEP, forts de leurs acquis expérientiels, tout en ayant une attitude prudente. Ils restent vigilants, car ils sont conscients que les risques existent comme dans toute activité professionnelle. Par ailleurs, ils énoncent des aspects de ressources qui pourraient être mobilisables pour la mise en œuvre du CEP. Ils s'interrogent sur les conditions de mise en œuvre du CEP. Pour deux tiers des conseillers interrogés, le CEP arrive à une période du parcours personnel et professionnel de transition, voire au moment d'un besoin de changement professionnel. Pour l'autre tiers, le CEP arrive à une période de leur parcours plutôt stable. L'auto-appréciation de leurs compétences à enrichir et/ou à compléter est globalement positive et ce moment de transition est vécu avec des perspectives positives en termes de projet d'évolution professionnelle, de renouvellement d'activité.

Soutiens: les soutiens désignent les aides apportées par autrui (famille, réseaux d'amis, réseaux professionnels...). Ces différents soutiens ont des modalités différentes: I'aide affective, l'aide à l'affirmation de soi, les encouragements et rétroactions, l'information, les conseils, les contacts utiles.

\section{La question proposée pour appréhender les soutiens est la suivante :}

Sur quelles ressources pouvez-vous compter pour apprivoiser cette nouvelle donne professionnelle?

«Mes ressources premières sont mes collègues»; «ma hiérarchie peut m'aider dans la démarche»; " l'institution à laquelle on appartient doit nous accompagner dans cette évolution»; «les réseaux professionnels dont les partenaires comme le Fongecif Ile-de-France, les OPCA, Pôle Emploi, Cap Emploi... sont des acteurs incontournables»; " les participants à ce groupe de formation en tant qu'acteurs de terrain»; «sur nos lieux de travail, les échanges en interne »; «les réunions de service»; "les contacts externes comme Défi métiers par exemple»; «aller au-delà du 10e arrondissement»; «les soutiens sont trouvés dans les formations réalisées et à réaliser»; «les proches sont des soutiens sans le savoir»; «ma famille est un tuteur pour moi»; «mes amis mes ressources quand j'ai des coups de blues»; «les ressources documentaires aident à rester en veille»; «mon service juridique m'a fourni pas mal d'informations sur cette nouvelle loi»; «mon meilleur soutien c'est moi».

L'auto-évaluation du soutien que l'on reçoit ou que l'on peut attendre lors d'une transition professionnelle est importante pour le sentiment de bien-être. Les conseillers sont en construction d'un espace de possibles, comme dans une démarche de 
construction de projet personnel et professionnel, pour envisager ce CEP dans leur pratique et dans leur contexte professionnels.

C'est l'entourage professionnel qui constitue le soutien le plus fortement exprimé pour apprivoiser cette nouvelle donne le CEP. Puis arrivent les échanges avec les collègues. Ces deux facteurs font partie intégrante du contexte de travail des conseillers et confortent l'idée évoquée par Nicholson (1984) que pour favoriser son adaptation professionnelle, le conseiller prend en compte son interaction «soi-organisation» pour faire le lien entre lui et son milieu de travail. Cette interaction peut être plus ou moins forte et/ou mettre l'accent sur l'un des deux pôles. Parmi les conseillers du corpus, certains s'inquiètent pour leur institution comme l'illustrent les propos suivants «avec l'arrivée du CEP, je crains pour l'avenir de mon institution»; «mon institution va-t-elle pouvoir s'adapter à ce virage à $180^{\circ}$, c'est un changement de prestation de service?».

Sans les citer, nous remarquons qu'en fonction des institutions d'appartenance les conseillers anticipent leur adaptation à ce changement différemment. Certains conseillers sont sur un mode d'absorption : leur institution est désignée pour dispenser le CEP et les conseillers envisagent de s'adapter en se conformant à son nouveau rôle et en assimilant les exigences induites par cette nouveauté; d'autres conseillers sont sur un mode de réplication : leur institution est désignée pour réaliser du CEP et les conseillers pensent qu'ils en font déjà donc envisagent de continuer d'agir comme avant. Sur le mode de la détermination, on trouve également les conseillers qui font partie d'une institution non désignée, donc avec un degré de liberté fort et qui estime déjà dispenser le CEP. Ils envisagent de s'adapter en fonction de leurs qualités, compétences et attentes. Enfin les conseillers qui envisagent leur adaptation sur le mode de l'exploration sont dans des institutions non désignées et le CEP est une nouveauté pour eux. Nous pouvons résumer en disant que la prise en compte du couple «acteur-situation » est essentielle pour comprendre les mécanismes de développement des conseillers.

Stratégie: les stratégies sont de trois sortes : celles visant à exercer un contrôle sur la situation; celles qui visent à contrôler la signification du problème et celles qui visent à gérer le stress.

Les questions proposées pour appréhender les stratégies sont les suivantes:

Comment vous y prenez-vous personnellement pour mener ce changement professionnel tout en gardant la main sur votre pratique? Comment faites-vous pour aborder sans trop de stress l'obligation qui vous est faite de vous mobiliser pour la mise en place du CEP?

\section{Les stratégies pour mener à bien ce changement...}

«En m'informant»; «en me documentant»; «en passant par des phases de formation comme aujourd'hui », «en échangeant avec les collègues et/ou avec des acteurs d'autres institutions »; «en suivant l'actualité dans centre Inffo»; «je suis aujourd'hui en attente de réponse de ma hiérarchie»; «je hiérarchise les priorités »; «je me fais mon propre projet personnel et professionnel »; «je m'adapte au fur et à mesure».

\section{Comment faites-vous pour aborder sans trop de stress l'obligation qui vous est faite de vous mobiliser pour la mise en place du CEP?}

«Je m'informe et reste en veille»; «je m'appuierai sur mes expériences précédentes»; "j'essaye de cerner les contours du CEP le plus possible»; «je reste attentive»; «je discute beaucoup avec les collègues!» «Pour moi ce CEP est une philosophie plutôt qu'une obligation »; «les choses se feront au fur et à mesure».

Les résultats relatifs à l'auto-évaluation de la stratégie envisagée par les conseillers montrent que tous les conseillers envisagent une stratégie pour mener à bien ce changement.

Ils ont chacun leur propre représentation du CEP et du coup l'envisagent à partir de registres différents.

Pour deux tiers des conseillers, ils envisagent des stratégies procédurales, à partir de leur répertoire de règles d'action correspondant à la base de leur métier. Cela s'exprime par des recherches d'outils, d'informations et de formations techniques. Cette forme de stratégie apparaît aussi très souvent à travers les attentes des conseillers formulées en début de formation. Ces attentes évoquent la recherche de la boîte à outils (les procédures, les bonnes recettes, les outils, les méthodes...) qui va permettre d'appréhender au mieux le CEP.

Ces stratégies procédurales sont basées sur des expériences déjà mobilisées par les conseillers et centrées sur la réussite de l'action dans l'immédiateté d'autant que la mise en œuvre du CEP doit se faire pour les conseillers désignés, via leur institution, à très court terme. 
Par ailleurs, pour un tiers des conseillers du corpus, ils envisagent d'emblée plutôt des stratégies analytiques qui sont centrées davantage sur la compréhension de l'action qu'ils vont devoir mobiliser. Ce type de stratégie se retrouve davantage chez les conseillers d'institutions non désignées d'office comme des opérateurs du CEP. Ces conseillers ont un questionnement davantage centré sur le «comment faire avec ma pratique actuelle de conseiller?».

\section{Discussion}

Globalement les conseillers envisagent la mise en œuvre du CEP sur la base de leurs expériences, en se faisant confiance. Chez les conseillers notamment qui ont appris sur le tas et par l'expérience, ils font, ils s'en sortent et ça marche. Ils ont une forme d'intelligence pratique, visant l'efficacité : «on bricole, on teste dans certaines situations, mais par des stratégies de contournement par exemple, on arrive à aider le sujet dans sa problématique d'orientation professionnelle ». À travers le dispositif réflexif proposé nous avons voulu solliciter les conseillers dans leur posture d'apprenant, c'est-à-dire dans une attitude qui présuppose qu'ils sont disponibles à tout apprentissage quelle que soit la situation et qu'ils soient favorables à l'«acte d'apprendre des situations». Par les exercices proposés, nous avons fait en sorte qu'ils prennent de la distance par rapport à leur action, qu'ils se mettent en posture de praticien réfléchi (Argyris et Shön, 1989) et de sujet capable de développer un regard sur leurs pratiques pour qu'ils s'adaptent plus rapidement à leur contexte de travail qui change.

Ce pari n'était pas gagné d'avance, car, en tant qu'animateurs de la formation, nous étions sans cesse rappelés sur le registre de la «réussite» de la coordination agie, alors que nous souhaitions les amener sur le registre des implications signifiantes: qu'est-ce qui fait sens pour vous? Que comprenez-vous du changement induit par le CEP?

Nous les avons sollicités à engager des questionnements pour qu'ils arrivent à transformer leurs objets de pensée (objets de travail) avec une double lecture : dans le réel, on trouve des actions matérielles, reliées par des relations de causalité; dans les représentations on trouve des opérations mentales, reliées par des relations d'implication signifiante. Notre hypothèse de travail est que c'est cette double lecture qui aide le conseiller à s'approprier son expérience comme une construction subjective à partir des actions qu'il réalise.

Cette liaison opérations-transformations permet de conjoindre la dimension fonctionnelle du processus (comprendre comment ça marche) et la dimension matérielle du processus (l'action des différents éléments les uns par rapport aux autres). Notre idée est de dire que la capacité à se représenter un processus matériel sous la forme d'un modèle mental donne à I'action une ampleur nouvelle, la met en perspective. Elle permet de mettre en œuvre une régulation proactive et met les conseillers dans une posture d'anticipation des événements avant qu'ils ne se produisent. Tel a été l'enjeu de ce travail.

Pour le conseiller en orientation et accompagnement professionnels, l'idée est de ne pas se centrer sur l'objet CEP, mais sur I'objet CEP mis en relation avec ses autres classes de situations de conseil déjà mobilisées; cela revient à penser les transformations et les changements dans le travail. Le principe d'éducabilité du sujet est au fondement du processus d'accompagnement professionnel.

Notre dispositif réflexif : aider les conseillers à repérer une ou plusieurs expériences formatives qui peuvent êtretransformées pour aller soutenir ce nouvel objet le conseil en évolution professionnelle est au cœur de ce principe d'éducabilité. Comment travailler l'image de soi dans un moment de transition professionnelle?

Cette approche développementale est à croiser avec l'approche interactionniste, car la transition en tant que processus de développement, comme nous l'avons évoqué, suppose de prendre en compte le nouvel environnement dans lequel va tendre le sujet et, qui impliquera pour lui la transformation de certaines valeurs, l'appropriation de nouveaux rôles et une modification de l'image de soi. Kaddouri (2006) parle de dynamiques identitaires qui renvoient au potentiel énergétique du sujet et qui intègrent un ensemble de tensions : tensions «entre » les différentes composantes de l'identité et tension «vers» un projet identitaire. L'orientation et les tensions qui orientent ce projet donnent lieu à des stratégies identitaires. «Vers quel métier me conduira ce CEP? Suis-je en mesure et en posture de le faire, de l'assumer?»

Même si les transitions sont à travailler à l'échelle de l'individu, elles renvoient à des collectifs de travail et à la manière dont les organisations et/ou institutions vont se saisir de ces mutations dans la sphère du travail. 
Travailler sur l'évolution professionnelle ne peut faire l'impasse sur les concepts convoqués : apprenance, expérience, transition, réflexivité, car ces derniers poussent à se retrouver en cohérence avec soi-même et pour soi-même : un point central de toute démarche de professionnalisation.

\section{Conclusion}

Le conseil en évolution professionnelle est un moment de transition professionnelle pour le conseiller en orientation et accompagnement professionnels, aussi envisagé comme une chance de développement de sa carrière, de son parcours et/ou comme un moyen de retrouver ou de renforcer ses valeurs. Les premiers résultats présentés confortent cette idée et apportent quelques éléments de compréhension sur la démarche de professionnalisation des conseillers rencontrés, même si certains conseillers expriment des craintes, inquiétudes, préoccupations, car ils ne sont pas tous sur les mêmes modes d'anticipation du changement. Le CEP est un moment de transition événementielle anticipée à laquelle chacun des conseillers va faire face. C'est un processus de changement professionnel qui a une envergure propre à chacun et que chacun vit différemment.

Concevoir le CEP comme un vecteur de transition professionnelle pour les conseillers c'est tenir compte que cette transition s'inscrit dans deux dimensions temporelles : celle de l'événement vécu au quotidien, mais aussi celle de l'empan très large d'une trajectoire de vie, en quelque sorte un maillage de deux temporalités. Ce mouvement de passage met en jeu des éléments existentiels et professionnels chez les conseillers, qu'ils ont pu exprimer dans le cadre du dispositif de formation — action réflexif proposé.

Ce travail nous a permis d'aborder l'arrivée du CEP dans une démarche exploratoire, à partir d'une conception développementale du sujet, d'aider les conseillers à mettre en relief leurs ressources par des questionnements suscitant l'auto-positionnement et à identifier les leviers pour faciliter et développer leur pratique et ainsi mettre en perspective leur ampleur professionnelle. Solliciter une attitude d'anticipation sur une pratique sans que les cadres ne soient posés.

L'analyse de l'activité constitue un détour incontournable pour éclaircir les questions des compétences et leur dynamique évolutive. On considère les compétences professionnelles du point de vue de leurs contenus spécifiques, des modalités et des conditions dans lesquelles elles se sont développées par les sujets individuels et des collectifs du travail.

Pour rendre compte de leur caractère dynamique, les compétences au travail doivent nécessairement être analysées d'un point de vue développemental. Ce développement est le produit d'un double processus endogène et exogène : par la formation ou la transmission de connaissances opérationnelles socialisées et historiquement élaborées, et par l'activité constructive propre de l'individu, car toute activité de travail a deux dimensions productive et constructive.

Notre défi a consisté à questionner les conseillers par le prisme de l'activité constructive afin qu'ils construisent et fassent évoluer leurs compétences en fonction de leurs situations et domaines professionnels d'action et qu'ils travaillent leurs expériences pour produire leurs compétences.

Pour terminer, un élément important mérite d'être mis en évidence concernant la dynamique de groupe créée pendant les sessions de formation. Les échanges interprofessionnels et interinstitutionnels ont facilité des points de rencontre entre les conseillers. Ce besoin de travailler tous ensemble a été fortement exprimé par l'ensemble des conseillers à chaque fin de session de formation. Il existe actuellement un cloisonnement entre les opérateurs désignés et plus globalement entre les acteurs de l'accompagnement professionnel et le CEP est une occasion de faire vivre l'idée du partage. «ll y a des enjeux de territoire, des secrets de fabrication propres à chacun, on aura besoin de partager». La dynamique de groupe engagée est très riche et est pointée comme un élément très positif par les conseillers. Cette dynamique les a interpellés sur la connaissance des partenaires, sur la manière dont ils devront travailler avec eux et mutualiser des ressources. Ces moments de travail ensemble ont aussi permis à certains d'entre eux de lever la barrière des représentations des institutions et de mieux appréhender les fonctions des conseillers d'autres institutions.

Par ailleurs la formalisation orale (groupe) et écrite (individuel), venue en soutien tout au long du processus de formation, a permis de produire ce que nous pouvons appeler des savoirs d'action favorisant ainsi le développement de nouvelles compétences collectives.

L'hypothèse de travail que nous ouvrons, et qui fera l'objet de nos travaux futurs, consiste à dire que le CEP est une occasion de reconstituer des collectifs de travail qui ont été souvent délaissés dans les institutions. Ces communautés de pratiques 
autour du métier du conseiller seraient envisagées comme une source de compétences où normes, règles de métiers, savoirs formels socialisés et informels pourraient être construits et partagés.

«Produire du sens dans une dynamique de socialisation professionnelle en te-

nant compte de la flexibilité du travail» (Poulin, 2015)

\section{Références bibliographiques:}

Ardoino, J. (1990). Les postures (ou impostures) respectives du chercheur, Vigneux, Matrice.

Argyris, C \& Schön, D. A (1989). Theory in practice: increasing professional effectiveness. San Francisco : Jossey Bass.

Balleux, A. \& Perez-Roux, T. (2013). «Transitions professionnelles», Recherche et formation, 74 (en ligne).

Boutinet, J-P. (2009). «Transition adulte », in ABC de la VAE, Toulouse : Erès, p.69-70

Carré, P. (2005). L'apprenance, vers un nouveau rapport au savoir, Paris, Dunod.

Cohen-Scali V. \& Guichard J. (2008). «L'identité : perspectives développementales», L'orientation scolaire et professionnelle, $n^{\circ} 27,3$, p. 321-345

Dewey, J. (1938). Expérience et éducation. Paris : A. Colin.

Elder, G.H Jr. (1994). "Trime, Human Agency and Social Change: perspectives on the Life Course", Social Psychology Quarterly, n 57 (1), p.4-15.

Jorro, A. (2011). L'expérience formatrice en formation professionnalisante. Communication au Colloque du REF. Louvain-LaNeuve, 14 septembre 2011.

Hoc, J.-M. (1990). La planification de l'activité. Dans J.-F. Richard, C. Bonnet et R. Ghiglione (s/d), Le traitement de l'information symbolique, traité de psychologie cognitive. Paris, Dunod/Bordas, (tome 2, p.223-228).

Jung, C. (1997). Types psychologiques. Paris, éditions Georg.

Kaddouri, M. (2006). «Dynamiques identitaires et rapports à la formation», in J.M. Barbier, E. Bourgeois, G. de Villers \& M. Kaddouri (dir), Constructions identitaires et mobilisation des sujets en formation, Paris : L'Harmattan, p.121-146.

Morrison, E.W. (1993). Newcomer information seeking: Explorating types, modes, sources, and outcomes. Academy of Management Journal, 36 (3), 557-589.

Leplat, J. (1991). Compétence et ergonomie. Dans R. Amalberti, M. de Montmollin et J. Theureu (s/d), Modèles en analyse du travail. Liège, Mardaga, p.263-278.

Nicholson, N. (1984). A theory of work role transitions. Administrative Science Quartely, 29 (2), p.172-191.

Pastré, P. (1997). Didactique professionnelle et développement. Psychologie Française, 42, 1; P. 89-100.

Perez-Roux T. \& Balleux A. (2012). «Transitions professionnelles choisies-imposées : quelles dynamiques identitaires à l'épreuve des contextes?» Congrès international AMSE, Reims 3-8 juin 2012.

Piaget, J. (1974). Réussir et comprendre. Paris, PUF.

Poulin, M. (2012). «Analyser sa trajectoire professionnelle par son récit de vie au travail ». Education permanente, $n^{\circ}$ Hors-série AFPA. - pp. 21-28

Poulin, M. (2015). «Le conseil en évolution professionnelle comme un vecteur de transition chez les conseillers AIOA» in Le conseil en évolution professionnelle (dir. P. Mayen). Passion éditions.

Rabardel, P. \& Duvenci-Langa (2004). «Dynamiques des compétences et dynamique des situations» in Recherches en didactique professionnelle (dir. R. Samurçay \& P. Pastré). Octares Editions.

Schwartz, Y. (2010). Quel sujet pour quelle expérience? Travail et apprentissages, nº 6. pp.11-25.

Schlossberg, N. K. (2005). «Aider les consultants à faire face aux transitions : le cas particulier des non-événements», L'orientation scolaire et professionnelle, 34/1 p. 85-101.

Vygotski, V-L. (1997). Pensée et langage. Paris : La Dispute.

Autres:

Arrêté du 16 juillet 2014 fixant le cahier des charges relatif au conseil en évolution professionnelle prévu à l'article L. 6111-6 du code du travail (NOR : ETSD1414403A Version consolidée au 19 avril 2015). 